\title{
Actor Positioning Based on Molecular Geometry in Aerial Sensor Networks
}

\author{
Mustafa İlhan Akbaş, Gürkan Solmaz and Damla Turgut \\ Department of Electrical Engineering and Computer Science \\ University of Central Florida \\ Email: \{miakbas,gsolmaz,turgut $\} @$ eecs.ucf.edu
}

\begin{abstract}
Advances in unmanned aerial vehicle (UAV) technology and wireless sensor and actor networks (WSAN) made it possible to equip small UAVs with sensors and deploy aerial sensor and actor networks. Aerial sensor networks enable high quality observation of events while reducing the number of requirements. Positioning of UAVs with actor nodes is critical in these systems for effective data collection.

In this paper we propose an actor positioning strategy for aerial WSANs considering the scenario of toxic plume observation after a volcanic eruption. The positioning algorithm utilizes the Valence Shell Electron Pair Repulsion (VSEPR) theory of chemistry, which is based on the correlation between molecular geometry and the number of atoms in a molecule. The limitations of the basic VSEPR theory are eliminated by extending the approach for multiple central data collectors. The simulations show that the proposed system provides high connectivity and coverage for the aerial sensor network.
\end{abstract}

\section{INTRODUCTION}

Wireless sensor and actor networks (WSANs) [1] consist of a large number of sensor nodes with limited capabilities and a smaller number of actors, which process the collected information and react accordingly. The recent advances in unmanned aerial vehicles (UAVs) with built in sensors made it possible to deploy autonomous aerial sensor and actor networks. UAV systems are cost effective and attractive solutions for surveillance applications and there are emerging concepts for employing autonomous UAV systems.

The scenario considered in this paper is a volcanic eruption such as the eruptions of the volcano Eyjafjallajökull at Eyjafjöll in Iceland in the Spring of 2010. The erupting lava of Eyjafjallajökull injected a cloud of ash into the Jet Stream. Even when the ashcloud was carried away from the volcano with the wind, it was not possible to bring personnel for close-up observation of the volcano or the plume. The UAV system we present here consists of small UAVs with sensor nodes and larger actor UAVs collecting the data. This system enables high quality observation of toxic plume behavior and provides a unique three dimensional system for environmental monitoring. In such a system, UAVs with actors, acting as central data collectors, can improve network performance in terms of energy consumption or traffic load balancing.

The dynamic positioning of the actors in three dimensional space is critical for effective coverage of the 3-D space and data collection. In this paper, an actor positioning strategy for aerial WSANs is presented to achieve these objectives while preserving 1-hop connectivity from each actor UAV to the central UAV in unprecedented settings of the scenario. The actors use a lightweight and distributed algorithm based on the Valence Shell Electron Pair Repulsion (VSEPR) theory to

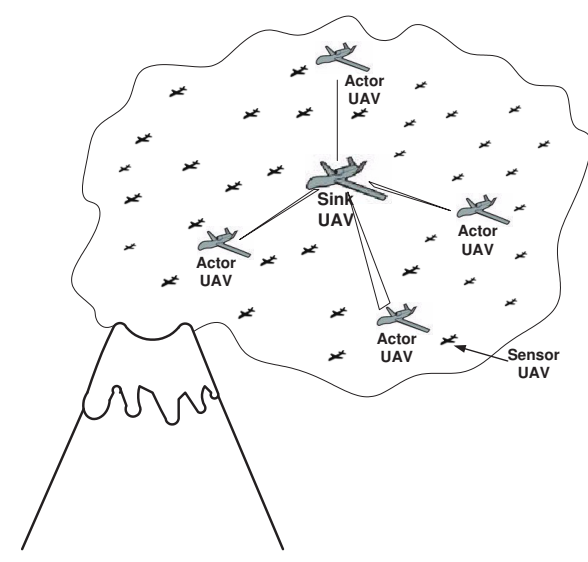

Fig. 1. Volcanic plume application scenario.

form a self organizing actor network. VSEPR theory is originally used in chemistry for the prediction of peripheral atom alignments around a central atom. This concept is adopted to define the rules of the algorithm designed to determine the actor positions. The basic VSEPR theory has limitations on the number of central and surrounding nodes. These limitations are eliminated by extending the adopted theory for multiple sinks to improve the scalability of the approach.

The rest of the paper is organized as follows. Section II summarizes the related work. We provide a detailed description for our approach in Section III. We show the simulation results in Section IV and finally conclude in Section V.

\section{RELATED WORK}

Although there is an increasing interest in applications of sensor networks in three dimensional (3D) space such as space exploration or airborne surveillance, most of the literature on dynamic node positioning strategies is limited to two dimensional (2D) space. The strategies designed for two dimensions become NP-hard in 3D space. The optimization strategies for 3D setups mostly focus on coverage problems. In the conventional 2D scenarios, the maximal coverage problem is mapped to a circle packing formulation which has a polynomial time solution. This problem turns into the sphere packing problem in three dimensions. Ravelomanana [2] studies the properties of network topologies that result from random deployment of nodes in a 3D region of interest to provide theoretical bounds. The author derives conditions for the node transmission range $r$ required for achieving a degree of connectivity $d$, where every node has at least $d$ 
neighbors. Pompili et al. [3] use Ravelomanana's bounds to validate the effectiveness of their 3D random underwater node deployment scheme. Alam and Haas [4] argue that space filling polyhedrons would be more suitable to use for 3D coverage and try to fill the 3D application space with the least number of polyhedrons for maximal coverage. Zhou et al. [5] present two algorithms for discovering boundary nodes and constructing boundary surfaces in 3D wireless networks. Bai et al. [6] designed and proved the optimality of one and two connectivity patterns under any value of the ratio of communication range over sensing range, among regular lattice deployment patterns. Slab Routing by Chiang and Peng [7] adapts $2 \mathrm{D}$ geographic face routing techniques to $3 \mathrm{D}$ space by dynamically creating a space partition and executing face routing over the planar projected graph of nodes contained within.

While there are aerial sensor network implementations for various applications, only a few of them focus on volcanic gas sampling. The autonomous aerial system by Astuti et al. [8] has a single UAV, which performs aerial surveillance of volcanic areas and analysis of the composition of gases inside volcanic plumes. The SensorFly system [9] by Purohit and Zhang is a mobile-controlled flying sensor network designed to monitor changes in dangerous environments. Elston and Frew [10] present a hierarchical control architecture with a mothership, a distributed database, and daughter micro air vehicles, which use vector field tracking. Autonomous Flying Robot MARVIN (Multipurpose aerial robot vehicles with intelligent navigation) project [11] uses aerial robots with the ability to coordinate with each other to complete required tasks.

VSEPR theory was first presented by Sidgwick and Powell [12] and refined by Gillespie and Nyholm [13]. The elaborate VSEPR theory states that the maximum repulsion of the electron pairs defines the geometric optimum positions of peripheral atoms that maximizes the distance between the electron pairs.

\section{Positioning MOdel}

\section{A. System model}

The system consists of $N$ nodes, with a set, $S$, of small UAVs, which have built-in sensor nodes and a set, $A$, of more powerful UAVs with actor nodes. There are also sinks, number of which depends on the geometry.

The formation of actor-sink backbone and affiliation of sensor nodes with the actors are similar to SOFROP [14]. The nodes do not follow any predetermined initial configuration. Each sensor node $s$ communicates only with direct neighbors $\operatorname{Neigh}(s)$ and keeps a "weight" value, which is " $k$-(hop value)" where $k$ is the weight of the actor and hop value is the number of hops required to reach the affiliated actor. The sensor nodes and actors are assumed to have transmission radii $r_{s}$ and $r_{a}$, respectively, with spherical transmission ranges.

\section{B. "VSEPR theory" approach}

The VSEPR model is the most successful model for the prediction of closed-shell molecule geometries. Laplacian of the charge density provides the physical basis for the VSEPR model. The spherical surface on which the electron pairs are assumed to be localized is identified with the sphere of

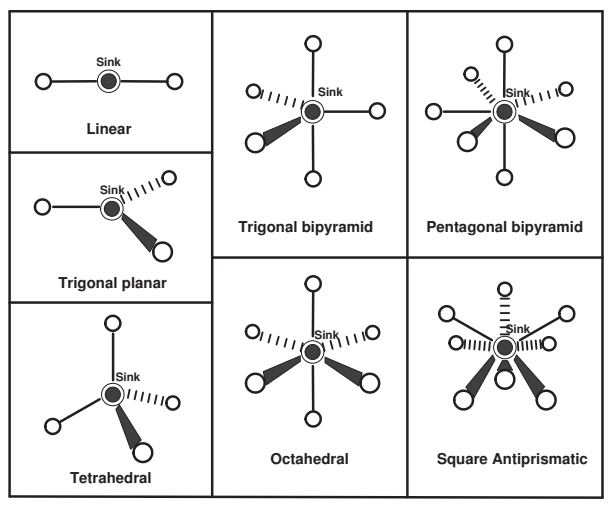

Fig. 2. VSEPR theory geometries.

maximum charge concentration in the valence-shell charge concentration and the localized pairs of electrons are identified with the local maxima.

VSEPR theory uses the "AXE method" of electron counting, in which $A$ is the number of central atoms, $X$ is the number of sigma bonds between the central atom and the surrounding atoms and $E$ is the number of lone electron pairs. The geometry predictions depend on the steric number, which is the sum of $X$ and $E . E$ is used particularly for deciding the positions of the actors in systems with multiple sinks in our approach.

VSEPR theory is applied such that the possible actor positions for different number of actors are found according to VSEPR theory geometries, which are given in Fig. 2. Then they are converted into positions with respect to the sink. The algorithm provides self organization of the system both for increasing and decreasing number of actors.

\section{VSEPR theory geometries of WSAN}

The geometries formed using the VSEPR theory are identified according to a reference point. The position of the sink, $p_{S}$, is taken as the reference origin in $X Y Z$ coordinate system during the flight and all other positions are calculated with respect to this origin. The formulation of geometries is critical for the definition of positions that the actors can be located and for the definition of transitions between geometries. The main direction that the UAV system headed on a time instant forms the $x$-coordinate and the positions of the actors are formulated according to this system.

When there are two actors, they are arranged in a "Linear" geometry, with an expected connection angle of $180^{\circ}$ and following positions:

$p_{a_{1}}(x, y, z)=(r, 0,0) \quad p_{a_{2}}(x, y, z)=(-r, 0,0)$

The geometrical model used when there are three actors around the sink is "Trigonal planar", in which the actors are in the following positions with connection angles of $120^{\circ}$.

$$
\begin{aligned}
& p_{a_{1}}(x, y, z)=(r, 0,0), \quad p_{a_{2}}(x, y, z)=\left(-r \cdot \sin \left(30^{\circ}\right), r \cdot \sin \left(60^{\circ}\right), 0\right) \\
& p_{a_{3}}(x, y, z)=\left(-r \cdot \sin \left(30^{\circ}\right),-r \cdot \sin \left(60^{\circ}\right), 0\right)
\end{aligned}
$$

When there are four peripheral actor UAVs, the sink is located at the center with four substituents that are located at the corners of a tetrahedron with connection angles of $\cos ^{-1}\left(\frac{-1}{3}\right) \approx 109.5$. 
$p_{a_{1}}(x, y, z)=(0,0, r), p_{a_{2}}(x, y, z)=(-r . a,-r . b, r \cdot \cos (109.5))$

$p_{a_{3}}(x, y, z)=\left(-r . \sin \left(109.5^{\circ}\right), 0, r \cdot \cos (109.5)\right)$

$p_{a_{4}}(x, y, z)=(-r . a, r . b, r . \cos (109.5))$

where $a=\sin \left(109 \cdot 5^{\circ}\right) \cdot \sin \left(30^{\circ}\right), b=\sin \left(109 \cdot 5^{\circ}\right) \cdot \cos \left(30^{\circ}\right)$

When there are five actors, they take positions with nonidentical connection angles. Three actors are positioned on the $y=0$ plane with connection angles of $120^{\circ}$ whereas the other two actors take positions on $y$-axis with angles of $90^{\circ}$ to the $y=0$ plane. Hence this geometry is a Trigonal bipyramid.

$p_{a_{1}}(x, y, z)=(r, 0,0), p_{a_{2}}(x, y, z)=\left(-r \cdot \sin \left(30^{\circ}\right), r \cdot \sin \left(60^{\circ}\right), 0\right)$

$p_{a_{4}}(x, y, z)=(0,0, r), p_{a_{3}}(x, y, z)=\left(-r \cdot \sin \left(30^{\circ}\right),-r \cdot \sin \left(60^{\circ}\right), 0\right)$

$p_{a_{5}}(x, y, z)=(0,0,-r)$

When there are six actors, they are arranged around the sink symmetrically, defining the vertices of an octahedron.

$$
\begin{array}{ll}
p_{a_{1}}(x, y, z)=(r, 0,0) & p_{a_{2}}(x, y, z)=(0, r, 0) \\
p_{a_{3}}(x, y, z)=(r, 0,0) & p_{a_{4}}(x, y, z)=(0,-r, 0) \\
p_{a_{5}}(x, y, z)=(0,0, r) & p_{a_{6}}(x, y, z)=(0,0,-r)
\end{array}
$$

The pentagonal bipyramid (or dipyramid) is a molecular geometry with one atom at the center with seven ligands at the corners of a pentagonal dipyramid.

$p_{a_{1}}(x, y, z)=(r, 0,0), \quad p_{a_{2}}(x, y, z)=\left(r \cdot \cos 72^{\circ}, r \cdot \sin 72^{\circ}, 0\right)$

$p_{a_{4}}(x, y, z)=(0,0, r), \quad p_{a_{3}}(x, y, z)=\left(-r \cdot \cos 36^{\circ}, r \cdot \sin 36^{\circ}, 0\right)$

$p_{a_{7}}(x, y, z)=(0,0,-r), \quad p_{a_{5}}(x, y, z)=\left(r \cdot \cos 72^{\circ},-r \cdot \sin 72^{\circ}, 0\right)$

$p_{a_{6}}(x, y, z)=\left(-r \cdot \cos 36^{\circ},-r \cdot \sin 36^{\circ}, 0\right)$

According to the VSEPR theory, the square antiprism is the favored geometry among the possible geometries with eight surrounding atoms. A square anti-prism corresponds to the shape when eight points are distributed on the surface of a sphere.

$$
\begin{array}{ll}
p_{a_{1}}(x, y, z)=\left(r \cdot a \frac{\sqrt{2}}{2}, 0, r \cdot \frac{h}{2}\right) & p_{a_{2}}(x, y, z)=\left(0, r . a \frac{\sqrt{2}}{2}, r \cdot \frac{h}{2}\right) \\
p_{a_{3}}(x, y, z)=\left(-r . a \frac{\sqrt{2}}{2}, 0, r \cdot \frac{h}{2}\right) & p_{a_{4}}(x, y, z)=\left(0,-r \cdot a \frac{\sqrt{2}}{2}, r \cdot \frac{h}{2}\right) \\
p_{a_{5}}(x, y, z)=\left(r . a, r . a,-r \cdot \frac{h}{2}\right) & p_{a_{6}}(x, y, z)=\left(-r . a, r . a,-r \cdot \frac{h}{2}\right) \\
p_{a_{7}}(x, y, z)=\left(-r . a,-r . a,-r \cdot \frac{h}{2}\right) & p_{a_{8}}(x, y, z)=\left(r . a,-r . a,-r \cdot \frac{h}{2}\right)
\end{array}
$$

where $a$ and $h$ are constants used in pentagonal bipyramid geometry to simplify the transitions. $h / 2 \approx 0.5237$ represents the positive and negative $z$ values for the planes that the actors are located at and $a \approx 1.2156$.

\section{Extension of VSEPR theory approach}

Initial VSEPR theory was presented for one central atom and at most eight surrounding atoms. Adoption of this theory allowed the design of at most eight actor UAVs and larger number of small UAVs. There are no current applications of UAV systems in practice with more data collector UAVs. Therefore APAWSAN [15] employed only the basic VSEPR theory for actor positioning. However this property of VSEPR theory introduces several limitations such as the number of actors and the central data collector UAV. Hence as additional contributions with respect to APAWSAN [15], an extended version of VSEPR theory is utilized to allow implementation of more than eight actors and multiple sinks.

R. J. Gillespie [16] presented application of VSEPR theory for twelve surrounding nodes around a central node, depending on same physical rules. Our system is extended by employing this approach. The favored geometries for nine to twelve actors, shown in Fig. 3, are tricapped trigonal prism, bicapped

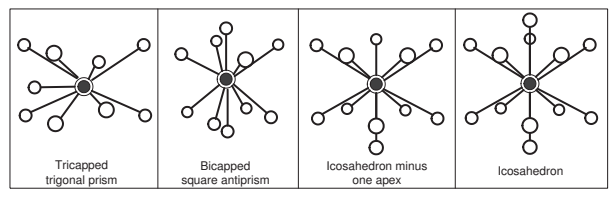

Fig. 3. Geometries of nine to twelve actors.

square antiprism, icosahedron minus one apex and icosahedron.

R.J. Gillespie also showed that molecules containing three or even four-center bonds conform to the general rule that the electron pairs around any central atom keep as far apart as possible. As another extension of VSEPR theory based method in our system, the limitation of basic VSEPR theory on scalability is removed by using multiple sink nodes. These sink nodes correspond to the multiple central atoms in the molecular geometries with .

Kettle [17] showed that the usual molecular orbitals which are used to describe the bonding in the metal cluster may be transformed into the localized two-center and three-center molecular orbitals described by VSEPR theory. When there are more than twelve actors, our system requires multiple sinks to form the actor geometries. Therefore the requirement of our approach is the deployment of more than one sink as the number of actors exceeds twelve. However the number of actors is already critical for sinks since the actors are actuators of the data collected by the sensor nodes and the data load on the sink increases as the number of actors per sink increases.

The positioning strategy of multiple sinks follows the same rules as the actor geometries, but without a central node. For instance, if there are six sinks in an aerial WSAN, they are positioned as the vertices of octahedral without a central node. An example of multiple sink geometries is given in Fig. 4. Four sinks form the tetrahedral geometry with an actor connected to each sink. The sinks are positioned according to the VSEPR theory rules such that each one forming tetrahedral geometries with three sinks and an actor.

\section{E. Dynamic positioning}

Our dynamic application scenario includes various requirements and challenges because of its differentiating features such as the lack of human control or the continuous motion during the flight. Therefore the geometries must not be very sensitive to small changes in positions and the actors must be able to reorganize in case of a change in the number of actors. The positioning algorithm is given in Algorithm 1.

In Algorithm 1, the common properties of calculated positions of actors in the specific geometries are used. All actor position options according to Algorithm 1 are on $z=0$ plane when $n$ is smaller than four with the condition $\Theta=$ $\frac{360}{n}$. When $n$ is equal to four, the geometry is perfectly

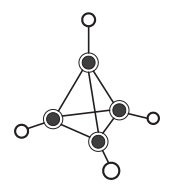

Fig. 4. An example of multiple sink geometries. 


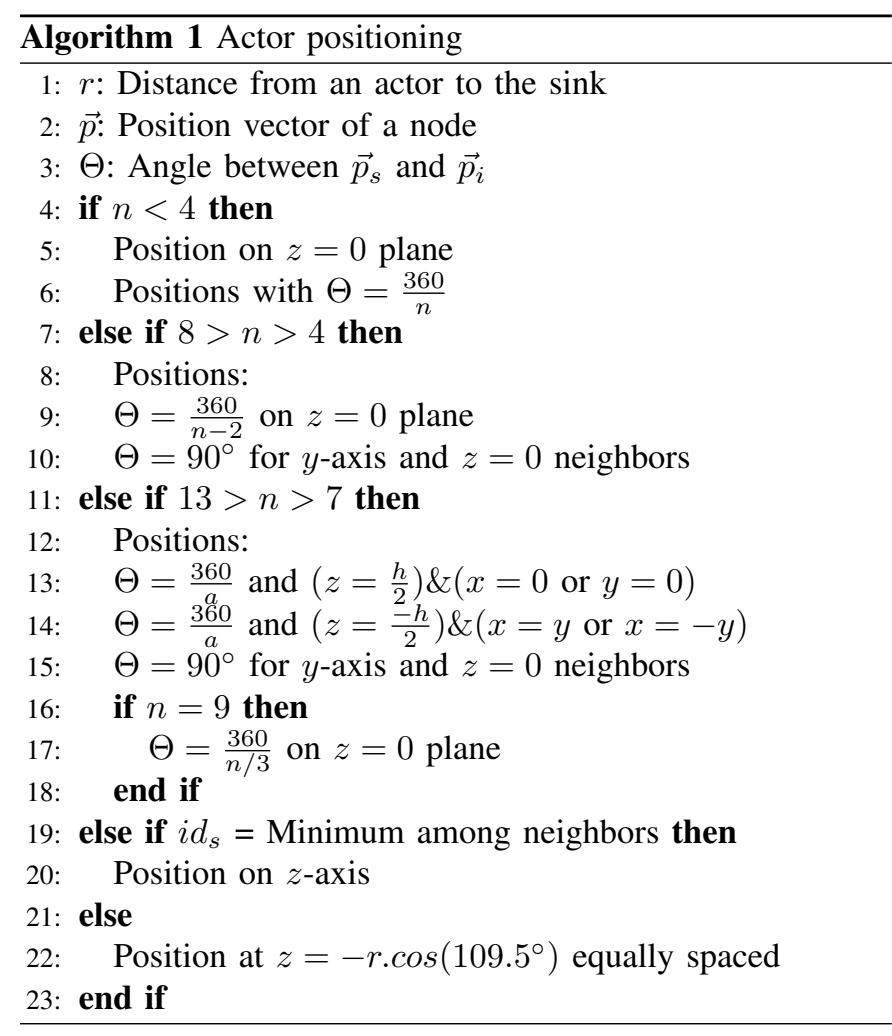

symmetrical. Therefore, the actor closest to $z=r$ plane takes $(0,0, r)$ position and all the other nodes are located with equal distances to each other. When $n$ is between four and eight, there are two actors on $z$ axis and the others are on $z=0$ axis plane. When the number of actors is larger than seven, the actors are positioned at least on two planes instead of one with a certain $z$ value.

\section{Simulation Study}

\section{A. Simulation environment}

The evaluation of the proposed system is conducted in OPNET modeler [18] by extending the node models created in SOFROP [14]. The transmission radius of a node is taken as 40 meters. There are 30 sensor nodes with IEEE 802.11 MAC layer and random mobility profile.

\section{B. Simulation results}

1) Experiment 1: There must be at least one actor in a sensor node's transmission range, which makes the coverage of the network backbone important for the system. For the calculation of the union volume of actor coverages, the inputs are number of spherical coverage volumes, coordinates of the actors, the reception range and expected memory usage by matrix used for modeling spheres. The union volume of actor coverages is calculated by a numerical approach, which first finds the most distant point in the coordinate system. Then the real coordinate system is projected to a boolean 3D matrix. The boundary points are found for each sphere and points fitting into the sphere are used to calculate the final volume.

Fig. 5 shows coverages for geometries with one sink and two sinks. As the number of actors increases up to nine, the coverages of the basic VSEPR theory geometries increase. However it can be observed that the bicapped square antiprism,

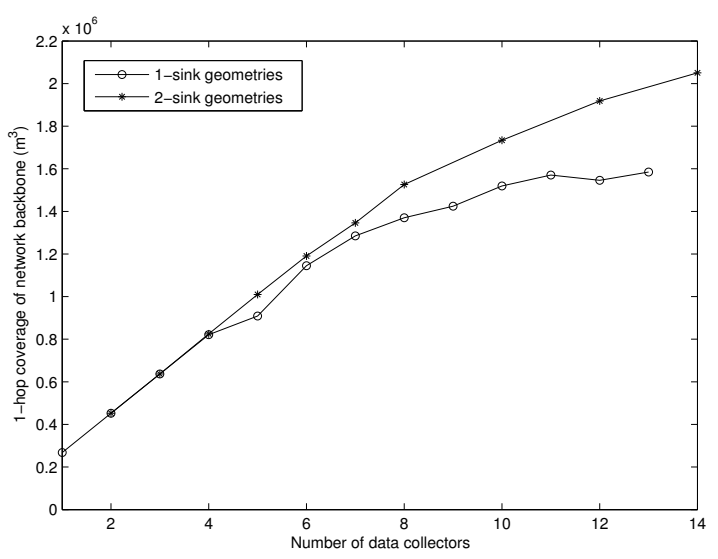

Fig. 5. 1-hop coverage for different geometries.

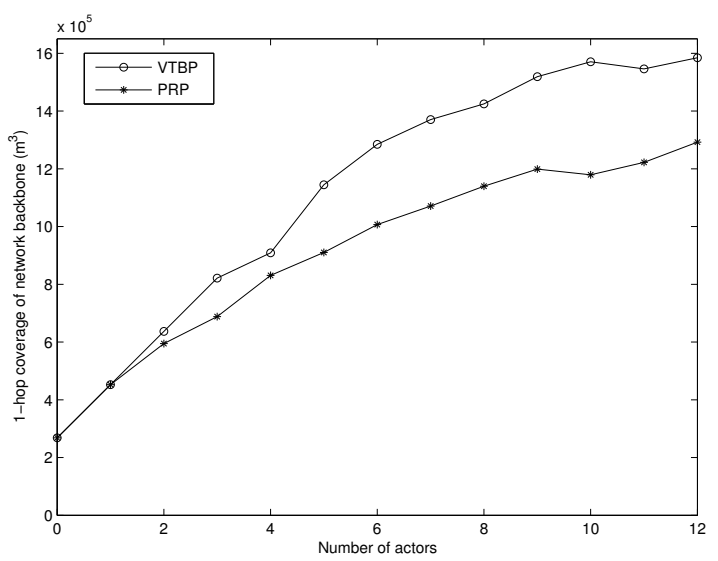

Fig. 6. 1-hop coverage for our protocol vs. random positioning with 1 sink.

icosahedron minus one apex and icosahedron are not as effective as the geometries with less actors. Additionally, it is observed that the coverages of 1-sink and 2-sink geometries are similar unless the number of data collectors exceeds seven. Therefore, the number of sinks must be increased to change the geometry of the actors for a more effective coverage when the number of actors exceeds seven.

2) Experiment 2: In the second set of experiments, the coverage of the proposed VSEPR theory based positioning(VTBP) approach is compared to a partially random positioning (PRP) method. PRP method is designed such that it includes the same number of the sink nodes for each geometry to compare and each actor node is at the same distance to retain the properties of network structure. Fig. 6 and Fig. 7 show the coverage for one and two sink geometries of both methods, respectively. The better coverage characteristic of our method is observed in both cases and the performance difference becomes higher as the number of actors increase.

3) Experiment 3: The maximum and the minimum hop number of the sensor nodes must not vary among actor areas in a network where the sensor nodes are shared efficiently among actors. Fig. 8 shows the maximum and the minimum weight values averaged over the nodes for all basic geometries. It can be observed that as the geometries evolve, the average minimum weight value increases and the range of weight values that the nodes acquire becomes smaller. The performance of the system improves considerably from the trigonal planar 


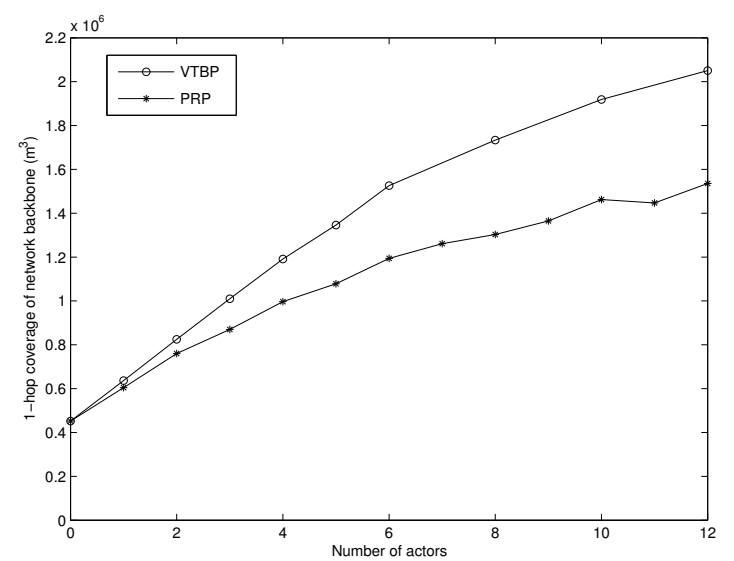

Fig. 7. 1-hop coverage for our protocol vs. random positioning with 2 sinks.

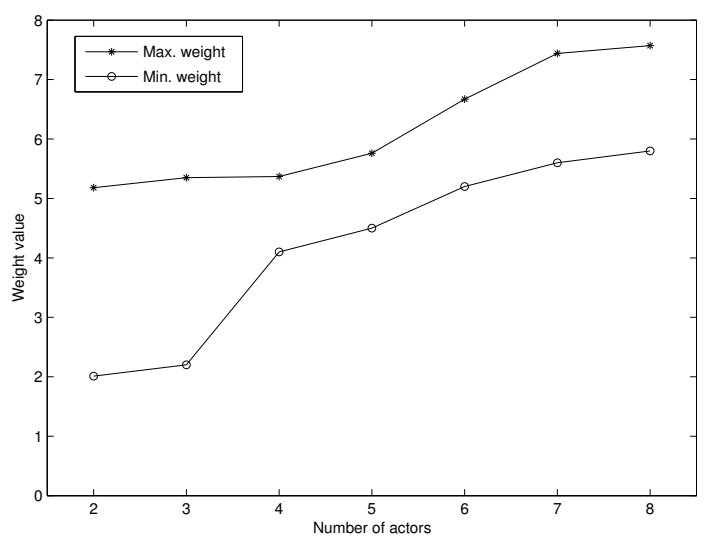

Fig. 8. Average maximum and minimum weight values for all geometries.

geometry to tetrahedral geometry.

4) Experiment 4: While using multiple actors, the concurrency becomes essential for an effective utilization of the system. Therefore cardinality is chosen as the metric to evaluate the performance of the system in distributing the actor affiliations. The fluctuations in the average cardinality of the actors are shown in Fig. 9. Higher fluctuation is a result of unequal distribution of the nodes to the actors during the lifetime of the network. Fig. 9 shows that the variation in the actor cardinalities decreases as the number of actors increases.

\section{Conclusion}

In this paper, a EXTEND actor positioning algorithm in aerial WSANs is introduced. The goal of the approach is to improve the on-site monitoring of the plume in a volcanic eruption scenario. The positioning algorithm utilizes VSEPR theory to overcome the challenges of the scenario. The basic rules of VSEPR theory are extended to overcome the limitation on the number of actors and only local communication is used for actor positioning. The experiments show that the system provides better coverage than random positioning while keeping 1-hop connectivity between each actor and sink. Future work includes the extension of the application of the molecular geometry to the actor positioning for large cluster of nodes and analyses for scalability and energy expenditure.

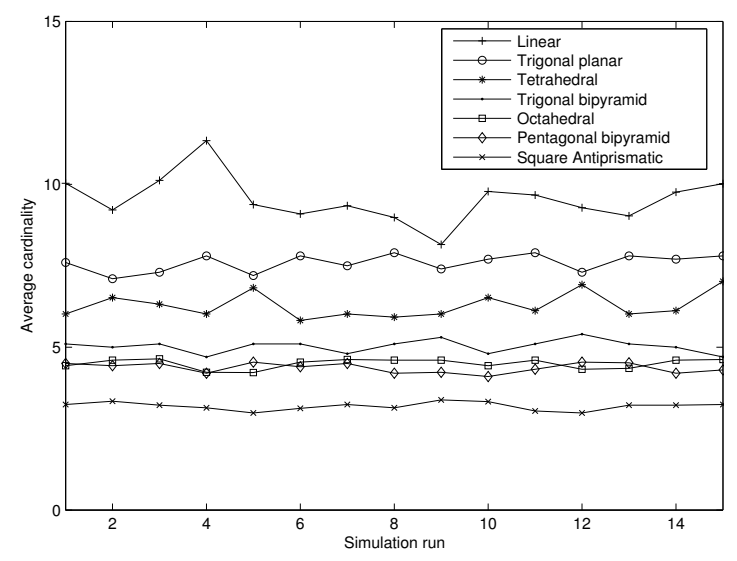

Fig. 9. Cardinality of actors for different geometries.

\section{ACKNOWLEDGEMENT}

The authors would like to thank OPNET Technologies Inc. for supporting this research by providing OPNET Modeler software under OPNET University Program.

\section{REFERENCES}

[1] R. Verdone, D. Dardari, G. Mazzini, and A. Conti, Wireless Sensor and Actuator Networks: Technologies, Analysis and Design. Academic Press, January 2008.

[2] V. Ravelomana, "Extremal properties of three-dimensional sensor networks with applications," IEEE Transactions on Mobile Computing, vol. 3, no. 3, pp. 246-257, July/September 2004.

[3] D. Pompili, T. Melodia, and I. F. Akyildiz, "Deployment analysis in underwater acoustic wireless sensor networks," in Proc. of WUWNeT, September 2006, pp. 48-55.

[4] S. M. N. Alam and Z. J. Haas, "Coverage and connectivity in threedimensional networks," in Proc. of MobiCom, September 2006, pp. 346357.

[5] H. Zhou, S. Xia, M. Jin, and H. Wu, "Localized algorithm for precise boundary detection in 3D wireless networks," in Proc. of International Conference on Distributed Computing Systems, June 2010, pp. 744-753.

[6] X. Bai, C. Zhang, D. Xuan, and W. Jia, "Full-coverage and kconnectivity $(\mathrm{k}=14,6)$ three dimensional networks," in Proc. of IEEE INFOCOM, April 2009, pp. 388-396.

[7] P. I.-S. Chiang and W.-C. Peng, "Slab routing: Adapting twodimensional geographic routing to three-dimensions," in Proc. of IEEE SECON, July 2009, pp. 1-9.

[8] G. Astuti, G. Giudice, D. Longo, C. D. Melita, G. Muscato, and A. Orlando, "An Overview of the 'Volcan Project': An UAS for Exploration of Volcanic Environments," J. Intell. Robotic Syst., vol. 54, no. 1-3, pp. 471-494, March 2009.

[9] A. Purohit and P. Zhang, "Sensorfly: A controlled-mobile aerial sensor network," in Proc. of SenSys, 2009, pp. 327-328.

[10] J. Elston and E. W. Frew, "Hierarchical distributed control for search and tracking by heterogeneous aerial robot networks," in Proc. of IEEE ICRA, May 2008, pp. 170-175.

[11] M. Dumiak, "Robocopters unite!" IEEE Spectrum, vol. 46, no. 2, p. 12, February 2009.

[12] N. V. Sidgwick and H. M. Powell, "Bakerian Lecture. Stereochemical Types and Valency Groups," Proc. R. Soc. A, vol. 176, no. 965, pp. 153-180, October 1940.

[13] R. Gillespie and R. Nyholm, "Inorganic stereochemistry," Quart. Rev. Chem. Soc., vol. 11, pp. 339-380, 1957.

[14] M. I. Akbas, M. R. Brust, and D. Turgut, "SOFROP: Self-organizing and fair routing protocol for wireless networks with mobile sensors and stationary actors," Elsevier Journal of Computer Communications, vol. 34, pp. 2135-2146, 2011.

[15] M. I. Akbas and D. Turgut, "APAWSAN: Actor positioning for aerial wireless sensor and actor networks," in Proc. of IEEE LCN, October 2011, pp. $567-574$.

[16] R. J. Gillespie, "The electron-pair repulsion model for molecular geometry," Journal of Chemical Education, vol. 47, no. 1, p. 18, January 1970.

[17] S. F. A. Kettle, Theoret. Chim. Acta, vol. 3, p. 211, 1965

[18] “OPNET Modeler," http://www.opnet.com. 